\title{
Relação entre exame clínico e radiográfico no diagnóstico da osteoartrite equina
}

\author{
Relationship between clinical and radiography examination for equine osteoarthritis \\ diagnosis
}

\author{
Raquel Yvonne Arantes BACCARIN ${ }^{1}$; Ana Paula Lopes de MORAES $^{1}$; Ana Carolina Rocha VEIGA ${ }^{1}$; Wilson \\ Roberto FERNANDES ${ }^{1}$; Marcos $\mathrm{AMAKU}^{2}$; Luis Claudio Lopes Correira da SILVA ${ }^{3}$; Stefano Carlo Filippo \\ HAGEN $^{3}$
}

${ }^{1}$ Departamento de Clínica Médica da Faculdade de Medicina Veterinária e Zootecnia da Universidade de São Paulo, São PauloSP, Brasil

${ }^{2}$ Departamento de Medicina Veterinária Preventiva e Saúde Animal da Faculdade de Medicina Veterinária e Zootecnia da Universidade de São Paulo, São Paulo-SP, Brasil

${ }^{3}$ Departamento de Cirurgia da Faculdade de Medicina Veterinária e Zootecnia da Universidade de São Paulo, São Paulo-SP, Brasil

\begin{abstract}
Resumo
A doença articular, especificamente osteoartrite, é uma das afecções mais prevalentes e debilitantes que acometem os equinos atletas. Apesar dos avanços tecnológicos nas últimas décadas, os exames clínico e radiográfico ainda são os meios mais comumente utilizados para o diagnóstico da osteoartrite equina. Neste estudo foram compilados e confrontados dados clínicos de 2872 equinos. Foram avaliados 146 casos de osteoartrite e analisadas radiograficamente 259 articulações com osteoartrite, para verificar quanto o exame radiográfico é condizente com o exame físico, e relacionar alterações clínicas com a modalidade de atividade física executada pelos cavalos. Pode-se constatar que a osteoartrite interfalangeana e metacarpo/metatarso falangeana (dígito) quando exibe alterações radiográficas faz com que os cavalos sejam mais propensos a claudicar, comparativamente a outros que também apresentam osteoartrite evidenciada radiograficamente, porém na articulação do tarso. Contudo, os escores radiográficos não correlacionaram a imagem radiográfica com a presença ou não de claudicação. A modalidade de atividade física não interferiu na frequência dos sinais clínicos de osteoartrite. Os equinos que mais apresentaram osteoartrite possuíam idade média de $8,4 \pm 3,9$ anos e eram utilizados para romaria, provas de quarto de milha e trabalho com o gado. Dentre as raças estudadas, as que mais frequentemente apresentaram equinos com osteoartrite foram Mangalarga Marchador, Crioulo e Quarto de Milha.
\end{abstract}

Palavras-chave: Osteoartrite. Claudicação. Equino. Radiografia. Atividade física.

\begin{abstract}
Joint disease, specifically osteoarthritis, is one of the most prevalent and debilitating diseases affecting athletic horses. Despite technological advances in recent decades, clinical and radiographic examinations are still the most commonly used methods for diagnosis of equine osteoarthritis. Clinical data of 2872 horses were compiled and compared for this study, it were evaluated 146 cases of osteoarthritis and radiographies of 259 affected joints were reviewed in order to verify how far radiographic examination is consistent with the clinical examination, and to correlate the clinical changes with physical activity performed by horses. Records showed that osteoarthritis in the fetlock and pastern joints (digit) when displaying radiographic changes makes horses more prone to show lameness, compared to others who also have osteoarthritis with radiographic evidence, but in the tarsocrural joint. However, radiographic scores do not correlate the radiographic image with the presence or absence of lameness. The type of physical activity performed by the horses had no influence on the frequency of clinical signs of osteoarthritis. The horses with osteoarthritis had an average of $8.4 \pm 3.9$ years old and were used for ride, western and work with cattle. Among the breeds studied, those that most frequently had horses with osteoarthritis were Mangalarga Marchador, Crioulo and Quarter Horse.
\end{abstract}

Keywords: Osteoarthritis. Lameness. Horse. Radiography. Physical activity.

\section{Correspondência para:}

Raquel Yvonne Arantes Baccarin

Universidade de São Paulo

Faculdade de Medicina Veterinária e Zootecnia (FMVZ)

Av. Prof. Dr. Orlando Marques de Paiva, 87. Butantã, São Paulo, SP
CEP.: 05508-270, Brasil

Fone/Fax: 5511 3091-1287/1283

e-mail: baccarin@usp.br

Recebido: 09/12/2010

Aprovado: 29/02/2012

Braz. J. Vet. Res. Anim. Sci., São Paulo, v. 49, n. 1, p. 73-81, 2012 


\section{Introdução}

Osteoartrite (OA) é uma das formas de afecção articular mais comum em humanos e animais de companhia, sejam equinos, cães ou gatos ${ }^{1}$. Estima-se que pelo menos $80 \%$ dos casos de claudicação e doenças articulares em animais são classificadas como $\mathrm{OA}^{2}$. Para equinos atletas é uma das afecções mais prevalentes e debilitantes.

Também chamada de doença articular degenerativa (DAD), a OA pode ter início a partir de diferentes fatores predisponentes, tais como trauma agudo ou crônico, sepse e osteocondrose, ou desenvolver-se como resultado de esforços repetidos em indivíduos que desempenham função atlética. A despeito da etiologia, a característica comum a todos estes processos é a degradação progressiva e lenta da cartilagem articular, que se torna adelgaçada e susceptível a fragmentação e destacamento, podendo ocorrer concomitantemente sinovite e efusão articular. Estas alterações caracterizam-se clinicamente por dor e disfunção da articulação ${ }^{3}$.

A dor, quando manifestada, indica que o limite de resistência da cartilagem foi ultrapassado. Levandose em consideração o fato da cartilagem articular ser desprovida de inervação, pode-se prever que quando os processos degenerativos eliciam dor, esses já atingiram certa profundidade na espessura desse tecido; eles são, dessa forma, inicialmente silenciosos, explicando o frequente atraso na detecção dessa enfermidade em seus primeiros estágios ${ }^{4}$.

Ainda hoje, apesar da possibilidade de cavalos que não claudicam possuírem sinais radiográficos compatíveis com OA, sabe-se que o diagnóstico de doença articular, como causa de uma claudicação, é na maioria das vezes baseado no exame radiográfico ${ }^{1}$. Alguns trabalhos também já sugeriram que não há relação entre alterações radiográficas, especificamente na articulação do tarso, e sinais clínicos ${ }^{5,6}$.

Frente a estas observações e sendo a OA uma importante causa de morbidade em equinos, pretendeu- se verificar o quanto o exame radiográfico é condizente com o exame físico, uma vez que são os principais métodos utilizados pelos clínicos para diagnóstico de OA equina, e, além disso, relacionar as alterações clínicas com o tipo de atividade física realizada por estes cavalos.

\section{Material e Método}

Durante um período de 10 anos, foram compilados dados clínicos de todos os equinos atendidos no Hospital Veterinário da Faculdade de Medicina Veterinária e Zootecnia da Universidade de São Paulo. Tais dados incluíram: raça, sexo, idade, peso, presença ou não de OA. Nos casos de cavalos diagnosticados com OA, foram compilados também os dados referentes a atividade exercida pelo animal, tempo de evolução da OA e o exame físico no dia do atendimento (presença de claudicação, dor palpável, efusão articular ou calor, sensibilidade de casco, sensibilidade muscular, teste de flexão articular).

Além disso, foi feita a avaliação das imagens radiográficas das articulações com OA, utilizando-se uma escala de 0 a 5 para cada um de nove itens similares ${ }^{7}$, ou seja: aumento de volume de tecidos moles periarticulares, mineralização de tecidos moles, aumento de espaço articular, diminuição de espaço articular, evidência de osteófitos, evidência de entesófitos, esclerose do osso subcondral, mudanças erosivas e evidências de fragmentos osteocondrais.

A análise estatística utilizou o teste do quiquadrado e o teste exato de Fisher para variáveis dicotômicas não pareadas e variável resposta dicotômica, e teste de Mann-Whitney para variável resposta ordinal (sendo dicotômico um dado com duas categorias e ordinal com mais de duas). Os três testes realizados foram não paramétricos. A análise foi realizada no Minitab e no StatXact-3 versão 3.11997.

O teste de Mann-Whitney foi utilizado para avaliação dos escores radiográficos das articulações acometidas. 


\section{Resultados}

No período de estudo, foram atendidos 2872 equinos acometidos por diferentes enfermidades, sendo 67\% machos e 33\% fêmeas. Deste total, 146 animais apresentavam OA (5\%), 96 machos (62\%) e 50 fêmeas (38\%), não havendo então diferença estatística entre as proporções de machos e fêmeas $(\mathrm{P}>0,05)$ (Tabela 1).

Dos 146 equinos com $\mathrm{OA}, 25 \%$ (36) não possuíam raça definida (SRD), 18\% (27) eram Quarto de Milha, 13\% (18) eram Mangalarga, 10\% (14) American Trotter e o restante de diferentes raças. No mesmo intervalo de tempo e computando-se todos os equinos examinados, o cavalo SRD (934) foi o mais frequentemente atendido (33\%), seguido por $23 \%$ de animais Mangalarga (645), 10\% de American Trotter (277) e 10\% de Quarto de Milha (275) (Tabela 1).
O tempo médio entre o início dos sintomas de OA e o atendimento hospitalar foi de $231 \pm 32$ dias (média \pm erro padrão).

Dentro da mesma raça, relacionando-se o número total de cavalos atendidos no período e o número de cavalos com OA, observou-se que 17\% dos animais da raça Mangalarga Marchador, levados ao hospital, apresentavam OA, 11\% dos animais da raça Crioula, 10\% dos Quarto de Milha, 10\% dos animais agrupados como cavalos de hipismo clássico (Sela Belga, Sela Holandesa e Sela Francesa), 7\% dos Apaloosa, 6\% dos Campolina, 6\% dos Puro Sangue Lusitano, 5\% dos Brasileiro de Hipismo, 5\% dos American Troter, 5\% dos Puro Sangue Inglês, 4\% dos SRD, 4\% dos Árabes, 3 \% dos Mangalarga, e nenhum da raça Bretã (Tabela 1).

Devido à variação do número de equinos examinados de cada raça, foram separados dois grupos de

Tabela 1 - Apresentação do número de equinos de cada raça atendidos no período, porcentagem (\%) das raças em relação ao total de equinos atendidos, número de equinos de cada raça com osteoartrite, porcentagem (\%) de equinos com $\mathrm{OA}$ de uma raça em relação ao número de equinos atendidos dessa raça, ao total de equinos com OA, e ao total geral de equinos atendidos no período - São Paulo - 2011

\begin{tabular}{|c|c|c|c|c|c|c|}
\hline \multirow[b]{2}{*}{ Raça } & \multicolumn{3}{|c|}{ Número de equinos atendidos } & \multicolumn{2}{|c|}{ Equinos com OA } & \multirow[b]{2}{*}{$\begin{array}{c}\% \text { em relação } \\
\text { ao total }\end{array}$} \\
\hline & $\mathrm{N}$ & $\begin{array}{l}\text { \% em relação } \\
\text { ao total }\end{array}$ & $\mathrm{N}$ & $\begin{array}{c}\text { \% em relação } \\
\text { ao N atendido } \\
\text { da raça }\end{array}$ & $\begin{array}{c}\text { \% em relação } \\
\text { ao total com } \\
\text { OA }\end{array}$ & \\
\hline Sem raça definida & 934 & 33 & 36 & 4 & 25 & 1,3 \\
\hline Mangalarga & 645 & 23 & 18 & 3 & 13 & 0,7 \\
\hline American Trotter & 277 & 10 & 14 & 5 & 10 & 0,5 \\
\hline Quarto de Milha & 275 & 10 & 27 & 10 & 18 & 0,9 \\
\hline Árabe & 161 & 6 & 7 & 4 & 5 & 0,2 \\
\hline Brasileiro de Hipismo & 156 & 5 & 8 & 5 & 5 & 0,3 \\
\hline Puro Sangue Lusitano & 129 & 5 & 8 & 6 & 5 & 0,3 \\
\hline Campolina & 77 & 3 & 5 & 6 & 3 & 0,2 \\
\hline Puro Sangue Inglês & 74 & 3 & 4 & 5 & 3 & 0,1 \\
\hline Mangalarga Marchador & 64 & 2 & 11 & 17 & 7 & 0,4 \\
\hline Apaloosa & 40 & 1 & 3 & 7 & 2 & 0,1 \\
\hline Grupo Sela* & 19 & 1 & 2 & 10 & 1 & 0,1 \\
\hline Crioulo & 18 & 1 & 2 & 11 & 1 & 0,1 \\
\hline Bretã & 3 & 0 & 0 & 0 & 0 & 0 \\
\hline TOTAL & 2872 & & 146 & & & \\
\hline
\end{tabular}

* Sela Francesa, Sela Belga, Sela Holandesa 
animais, ou seja, com maior porcentagem de animais com osteoartrite $(\mathrm{P}<0,05)$ (Crioulo, Quarto de Milha, Mangalarga Marchador e cavalos de hipismo clássico) e menor porcentagem (Apallosa, Árabe, American Trotter, Brasileiro de Hipismo, Campolina, Mangalarga, Puro Sangue Inglês, Puro Sangue Lusitano, SRD). Frente a esta disposição, não foi observada diferença na presença de OA entre as diferentes raças que compunham cada grupo $(\mathrm{P}>0,05)$.

A média da idade dos equinos sem $\mathrm{OA}$ foi de 7,3 \pm 4,5 anos e dos equinos com OA de $8,4 \pm 3,9$ anos, sendo significativa a diferença entre as idades $(\mathrm{P}<0,05)$. Também foi observado que os equinos com OA eram mais pesados $(420,45 \pm 73,6 \mathrm{~kg})$ do que os sem OA $(385,92 \pm 100,8 \mathrm{~kg})(\mathrm{P}<0,05)$.

Constatou-se que $95 \%$ dos cavalos com OA praticavam esporte ou atividade física intensa e somente $5 \%$ estavam ligados à reprodução. A principal atividade física exercida pelos cavalos com OA foi romaria, ou seja, cavalgada de longa distância com baixa intensidade, seguida pelas provas de quarto de milha e uso no trabalho com gado.

Independentemente do tipo de atividade física que exerciam, a maioria dos cavalos com OA (78\%) claudicou durante o exame físico, $61 \%$ apresentavam dor articular palpável, $41 \%$ presença de efusão articular ou calor, $32 \%$ sensibilidade de casco e $33 \%$ sensibilidade muscular.

Não houve relação entre a modalidade de atividade física exercida e o fato do cavalo com OA claudicar $(\mathrm{P}>0,05)$. Também não houve relação entre a modalidade de atividade física exercida com a presença de dor palpável, sensibilidade de casco, sensibilidade muscular, ou teste positivo de flexão articular $(\mathrm{P}>0,05)$. Porém, encontrou-se relação entre modalidade de atividade física e o fato destes cavalos apresentarem efusão e calor articular $(\mathrm{P}<0,05)$.

Também não foi possível relacionar a presença de claudicação com a presença de dor articular palpável, efusão articular ou calor, sensibilidade de casco e sensibilidade muscular $(\mathrm{P}>0,05)$.
Independentemente da articulação acometida pela OA, contatou-se que $39 \%$ dos cavalos apresentaram flexão positiva das articulações interfalangeanas, 71\% da articulação metacarpo/metatarsofalangeana, 12\% da articulação do carpo, 38\% da articulação do tarso, 9\% da articulação fêmur-tíbio-patelar.

Foram analisados os exames radiográficos de 259 articulações dos equinos acometidos por OA. Concomitantemente, foram fixados escores de acordo com o descrito no material e métodos. Além disso, foi relacionada a presença ou não de claudicação (Tabela 2).

As articulações do dígito (140) e do tarso (110) foram as principais articulações cuja OA exibiu alterações radiográficas. Analisando-se os membros sem claudicação, observou-se um número maior de articulações do tarso com OA (34) do que articulações do dígito com OA (21) e também com escore médio maior (tarso $=2,5 \pm 0,66$, dígito $=0,1 \pm 0,06$; média \pm erro padrão, $\mathrm{P}<0,01)$. Já nos membros apresentando claudicação, há um número maior de articulações do dígito com OA (119) do que de tarso (76) e desta vez com escores médios muito próximos entre elas, não diferindo estatisticamente (tarso $=2,1 \pm 0,44$, dígito $=$ $2,0 \pm 0,45, \mathrm{P}>0,05)$. O escore médio das articulações do dígito com claudicação foi maior do que o atribuído às sem claudicação (com claudicação $=2,0 \pm 0,45$; sem claudicação $=0,1 \pm 0,06 ; \mathrm{P}<0,05)$, o que não ocorre nas articulações do tarso, onde os escores médios são semelhantes, independentemente da presença de claudicação (com claudicação $=2,1 \pm 0,44$; sem claudicação $=2,5 \pm 0,66 ; \mathrm{P}>0,05)($ Tabela 2).

\section{Discussão}

$\mathrm{O}$ diagnóstico da $\mathrm{OA}$ equina é geralmente baseado em alterações clínicas e, principalmente, radiográficas, o que muitas vezes ocorre tardiamente. Apesar disto, o exame radiográfico ainda é a técnica por imagem mais utilizada mundialmente para diagnóstico de doenças articulares. O conhecimento de fatores 
Tabela 2 - Graduação em escore (0-45) das imagens radiográficas de 259 articulações de equinos acometidos por osteoartrite, segundo a presença de claudicação - São Paulo - 2011

\begin{tabular}{|c|c|c|c|c|c|}
\hline \multirow[b]{2}{*}{ Articulação } & \multirow{2}{*}{$\begin{array}{l}\text { Com OA } \\
\begin{array}{c}\text { Número } \\
\text { total }\end{array}\end{array}$} & \multicolumn{2}{|c|}{ Sem claudicação } & \multicolumn{2}{|c|}{ Com claudicação } \\
\hline & & $\begin{array}{l}\text { Número } \\
\text { de } \\
\text { articulações }\end{array}$ & $\begin{array}{l}\text { Intervalo de valor de } \\
\text { escore encontrado nas } \\
\text { diferentes articulações }\end{array}$ & $\begin{array}{l}\text { Número } \\
\text { de articulações }\end{array}$ & $\begin{array}{l}\text { Intervalo de valor de } \\
\text { escore encontrado nas } \\
\text { diferentes articulações }\end{array}$ \\
\hline interfalangeana distal & 31 & 5 & 0 a 1 & 26 & 0 a 8 \\
\hline interfalangeana proximal & 68 & 8 & 0 & 60 & 0 a 29 \\
\hline $\begin{array}{l}\text { Metacarpo/metatarso } \\
\text { falangeana }\end{array}$ & 41 & 8 & 0a 1 & 33 & 0 a 16 \\
\hline $\begin{array}{c}\text { Total de articulações dos } \\
\text { dígitos } \\
(\mathrm{M} \pm \mathrm{EPM})\end{array}$ & 140 & 21 & $(0,1 \pm 0,06) \mathrm{Aa}$ & 119 & $(2,0 \pm 0,45) \mathrm{Ab}$ \\
\hline carpo & 3 & 0 & - & 3 & 0 \\
\hline tibio-társica & 33 & 9 & 0 all & 24 & 0 a 8 \\
\hline intertarsiana & 36 & 12 & 0 a10 & 24 & 0 a 14 \\
\hline tarsometatarsiana & 41 & 13 & 0 a 9 & 28 & 0 a 15 \\
\hline $\begin{array}{l}\text { Total de articulações do } \\
\quad \operatorname{tarso}(M \pm E P M)\end{array}$ & 110 & 34 & $(2,5 \pm 0,66) \mathrm{Ba}$ & 76 & $(2,1 \pm 0,44) \mathrm{Aa}$ \\
\hline fêmur-tíbio-patelar & 6 & 0 & - & 6 & 0 a 4 \\
\hline TOTAL & 259 & 55 & & 204 & \\
\hline
\end{tabular}

$\mathrm{OA}=$ osteoartrite; $\mathrm{M}=$ média; $\mathrm{EPM}=$ erro padrão da média

Letras minúsculas: diferença estatística entre colunas; Letras maiúsculas: diferença estatística entre linhas.

predisponentes para o desenvolvimento da OA, como por exemplo, tipo de atividade física, poderia indicar quais cavalos necessitam de exames físicos mais precoces e frequentes para se prevenir o desenvolvimento e instalação desta enfermidade.
Neste estudo, a média de idade dos cavalos com OA foi 8,4 anos, compatível com adulto jovem, diferentemente do que ocorre em humanos, em que a $\mathrm{OA}$ é mais frequentemente encontrada no idoso. Aproximadamente metade das pessoas com mais de 65 anos 
apresenta sinais radiográficos de OA; sua prevalência aumenta para $85 \%$ nos com mais de 80 anos 8 . Já em cavalos atletas, a OA, devido a traumas repetitivos, é a causa mais comum de claudicação?.

O exercício é um fator predisponente para o desenvolvimento de $\mathrm{OA}$ em equinos, fato já confirmado por estudos experimentais e clínicos $6,{ }^{10}$, e que pode ser sugerido neste trabalho visto que $95 \%$ dos cavalos com OA praticavam algum tipo de esporte ou eram submetidos a exercícios de alta repetibilidade e intensidade.

Apesar dos cavalos SRD terem sido a maioria em atendimento, tanto para os acometidos por OA (37), como por outras enfermidades (897), a ocorrência de OA dentro dessa categoria foi muito pequena (4\%) comparativamente aos Mangalarga Marchador (17\%), Crioulos (11\%), e Quarto de Milha (10\%).

A ocorrência de $\mathrm{OA}$ entre os cavalos de hipismo clássico (Sela Francesa, Sela Belga e Sela Holandesa) foi $10 \%$, igualmente à porcentagem da categoria Quarto de Milha, contudo, deve-se considerar que esta porcentagem reflete o atendimento de somente dois casos.

As atividades físicas mais frequentemente exercidas pelos equinos que apresentavam $\mathrm{OA}$ foram romaria (32\%), provas de quarto de milha (tambor, baliza, laço, etc) (22\%) e de trabalho (17\%), o que coincide com as raças de cavalos que frequentemente praticam estas modalidades e que mais apresentaram $\mathrm{OA}$ no período de estudo, ou seja, SRD (25\%), Mangalarga (13\%) e Quarto de Milha (18\%). Alguns animais da raça América Trotter e da raça Mangalarga Marchador, cujas porcentagens de atendimento também foram maiores (10\% e $7 \%$ respectivamente), também eram utilizados para romaria.

A diferença encontrada entre o número de animais machos ou fêmeas com OA refletiu a tendência do atendimento no local, e não uma maior ocorrência de OA em machos, ou seja, no mesmo período de tempo também foi atendido um maior número de cavalos machos com outras enfermidades.
O longo tempo de $231 \pm 32$ dias entre o início dos sintomas e o atendimento clínico é explicado pelo fato dos cavalos acometidos levemente pela OA ainda serem utilizados quando os sinais clínicos são facilmente abolidos com o uso de anti-inflamatórios ${ }^{11}$.

Na literatura, um dos sinais clínicos mais comumente relatados pelos proprietários de cavalos com OA é a claudicação ${ }^{3}$, e realmente $78 \%$ dos cavalos com OA mostraram claudicação durante o exame físico. Contudo, não houve relação estatística entre claudicação e presença de dor palpável com o tipo de atividade física exercida pelo animal.

Interessante observar que houve um número maior de animais utilizados para provas de quarto de milha, romaria e para o trabalho que mostraram efusão articular ou calor $(\mathrm{P}<0,05)$. Persson ${ }^{12}$ observou que o movimento articular leva a um aumento do volume de líquido sinovial, provavelmente devido à maior difusão plasmática para o interior da cavidade articular. Esta afirmação poderia explicar a efusão articular nos cavalos praticantes das três modalidades citadas não fosse o fato dos outros esportes também promoverem grande amplitude de movimentação articular. Supõese então que a falta de adaptação e o treinamento irregular possam ter influenciado o aparecimento de efusão articular, principalmente, nos cavalos praticantes de romaria.

Todos os cavalos utilizados apresentaram alterações radiográficas de OA em alguma das articulações, e somente $78 \%$ mostraram claudicação, $61 \%$ dor palpável articular, $41 \%$ presença de efusão articular ou calor, mostrando que há certa relação entre sinais clínicos e aparência radiográfica. Alguns trabalhos referem que existe baixa ou modesta relação entre alterações radiográficas e presença de claudicação. Deve-se levar em conta que a maioria destes trabalhos foi realizada com OA társica e que os mesmos ressaltam falta de correlação entre a gravidade de claudicação e aspecto radiográfico e não quanto a presença ou não de claudicação $0^{5,10,13}$. Já no levantamento realizado por Garcia 
et al. ${ }^{6}$ somente $24 \%$ dos cavalos Mangalarga Marchadores com OA társica apresentaram claudicação.

Além disso, outro dado que dificulta o estabelecimento da OA como causa de uma claudicação em muitos casos, é que alguns cavalos mostram sensibilidade de casco e muscular concomitante a OA, ou seja, foi observado que $32 \%$ dos cavalos com OA mostraram sensibilidade de casco, e 33\% sensibilidade muscular.

Conforme McIlwraith ${ }^{4}$ a dor articular também pode ser detectada realizando-se o teste de flexão articular. Garcia et al. ${ }^{6}$ constataram que somente $16 \%$ dos potros que apresentavam alterações radiográficas de $\mathrm{OA}$ foram positivos ao teste de flexão articular. Esta falta de sensibilidade do teste também foi observada por Maranhão et al. ${ }^{13}$. Kawcak et al. ${ }^{10}$ observaram aumento na resposta ao teste de flexão articular em articulações saudáveis de cavalos após serem exercitados em esteira por 91 dias.

No presente estudo, a maioria dos animais submetidos ao teste de flexão das articulações metacarpo/ metatarsofalangeanas $(71 \%)$ mostrou resposta positiva, fato que não ocorreu em outras articulações. No caso da articulação do tarso, somente $38 \%$ dos cavalos foram positivos ao teste de flexão, o que coincide com as observações de Maranhão et al. ${ }^{13} \mathrm{e}$ Garcia et al. ${ }^{6}$.

Durante a análise dos escores radiográficos, notouse que existiam articulações sem alteração radiográfica localizada em membros sem claudicação, mas com OA presente. Nestes casos, foram encontrados dor e efusão articular e teste de flexão positivo, associados à diminuição de desempenho atlético, porém, sem claudicação e ainda sem alteração radiográfica visível, compatível com OA tipo 1, segundo McIlwraith ${ }^{3}$. A cartilagem articular não é vista radiograficamente exceto quando há uma extensa perda cartilagínea e diminuição do espaço articular; e 30 a 40\% de alteração na densidade mineral óssea são necessárias antes de alterações serem observadas ao exame radiológico, ou seja, esta falta de sensibilidade faz com que diagnósticos radiográficos precisos e precoces não possam ser realizados.

Mediante os resultados obtidos pela avaliação radiográfica das articulações com $\mathrm{OA}$, não foi possível relacionar o menor ou maior escore com a presença ou não de claudicação. Porém, se forem avaliadas somente as articulações interfalangeana distal, proximal e metacarpo/metatarsofalangeana, ou seja, do dígito, pode-se notar que os membros sem claudicação apresentaram escores menores na avaliação radiográfica $(\mathrm{P}<0,05)$.

Diferentemente ocorre com a articulação do tarso, onde membros sem claudicação mostram escores médios similares a membros com claudicação. Nesta articulação, reforçam-se as observações feitas por Hopper et al..$^{14}$; Maranhão et al. ${ }^{13}$; Bruyere et al. ${ }^{15}$; ByamCook e Singer ${ }^{5}$ e Garcia et al. ${ }^{6}$, de que há baixa correlação entre claudicação e a aparência radiográfica da articulação do tarso.

Segundo McIlwraith ${ }^{4}$, os pacientes que apresentam alterações radiográficas compatíveis com OA não necessariamente apresentam os sinais clínicos característicos da doença articular. Nos casos avançados de $\mathrm{OA}$, as articulações de baixa mobilidade, como as intertarsianas e tarsometatarsiana, podem se apresentar clinicamente sadias em relação a função e por outro lado apresentar anquilose óssea completa ${ }^{16,17}$.

Isto explicaria o fato dos cavalos deste estudo terem apresentado escores radiográficos similares nas articulações do tarso, e não apresentarem claudicação, pois somente a articulação tíbio-társica é considerada uma articulação de alta mobilidade ${ }^{18}$.

Kawcak et al ${ }^{10}$ mostraram forte correlação entre lise óssea no exame radiográfico e presença de claudicação, e entre proliferação óssea e quantidade de prostaglandina $\mathrm{E}_{2}$ no líquido sinovial. Também mostraram moderada correlação entre proliferação óssea e clau- 
dicação, e entre presença de osteofitos e resposta ao teste de flexão.

Segundo Charlotte et al. ${ }^{19}$, a articulação metacarpofalangeana é a mais comumente acometida pela OA em cavalos selvagens, ou seja, pela ocorrência natural. Em contrapartida, McIlwraith ${ }^{4}$ cita as articulações interfalangeana proximal e distais do tarso como as comumente acometidas por $\mathrm{OA}$ em animais idosos.

Neste estudo, a articulação interfalangeana proximal foi a mais acometida pela OA (68), seguida pela tarsometatarsiana (41) e metacarpo/metatarsofalangeanas (41), lembrando-se que a média de idade destes animais foi de $8,4 \pm 3,9$ anos, ou seja, não eram cavalos idosos.

\section{Referências}

1. MOBASHERI, A.; HENROTIN, Y. Identification, validation and qualification of biomarkers for osteoarthritis in humans and companion animals: Mission for the next decade. Veterinay Journal, v. 185, n. 2, p. 95-97, 2010.

2. LEE, P. Pharmacology of drugs used to treat osteoarthritis in veterinary practice. Inflammopharmacology, v. 11, n. 4, p. 385-399, 2003.

3. MCILWRAITH, C. W. Disease process of synovial membrane, fibrous capsule, ligaments and articular cartilage. In: STASHAK, T. S. Adam's lameness in horse. 4. ed. Philadelphia: Lippincott Williams \& Wilkins, 2002. p. 459-645.

4. MCILWRAITH, C. W. General pathobiology of the joint and response to injury. In: MCILWRAITH, C. W.; TROTTER, G. W. Joint disease in the horse. Philadelphia: W. B. Saunders, 1996. p. $40-70$.

5.BYAM-COOK, K. L.; SINGER, E. R. Is there a relationship between clinical presentation, diagnostic and radiographic findings and outcome in horses with osteoarthritis of the small tarsal joints? Equine Veterinary Journal, v. 41, n. 2, p. 118123, 2009.

6. GARCIA, R. S.; MELO, U. P. de; FERREIRA, C.; TOSCANO, F. S.; CRUZ, G. M. da. Estudo Clínico e radiográfico da osteoartrite társica juvenil em potros da raça Mangalarga Marchador. Ciência Animal Brasileira, v.10, n. 1, p. 254-260, 2009.

7. KIRKER-HEAD, C. A.; CHANDNA, V. K.; AGARWAL, R. K.; MORRIS, E.; TIDWELL, A.; O'CALLAGHAN, M. W.; RAND, W.; KUMAR, M. S. Concentrations of substance P and prostaglandin $\mathrm{E}_{2}$ in synovial fluid of normal and abnormal joints of horse. American Journal of Veterinary Research, v. 61, n. 6, p.714-718, 2000

8. PEDRINELLI, A. P.; GARCEZ-LEME, L. E.; NOBRE, R. S. A. $\mathrm{O}$ efeito da atividade física no aparelho locomotor do idoso. Revista Brasileira de Ortopedia, v. 44, n. 2, p. 96-101, 2009.

9. KAWCAK, C. E.; MCLLWARAITH, C. W.; NORRDIN, R. W.; PARK, R. D.; JAMES, S. P. The role of subchondral bone in

\section{Conclusões}

Alterações radiográficas discretas nas articulações do dígito devem ser consideradas como fonte de dor e, consequentemente, de claudicação em cavalos que desempenham atividade física, ao contrário das que ocorrem nas articulações do tarso. Em contrapartida, imagens radiográficas sem alterações devem ser interpretadas com cautela em cavalos que apresentam claudicação, para que o diagnóstico da $\mathrm{OA}$ não ocorra somente após a doença ter se estabelecido.

Dentre as diferentes modalidades de atividades físicas exercidas pelos cavalos deste estudo, as práticas de romaria, provas de quarto de milha e trabalho com o gado predispuseram mais frequentemente ao aparecimento de OA.

joint disease: a review. Equine Veterinary Journal, v.33, n. 2, p. 120-126, 2001.

10.KAWCAK, C. E.; FRISBIE, D. D.; WERPY, N. M.; PARK, R. D.; MCLLWRAITH, C. W. Effects of exercise vs experimental osteoarthritis on imaging outcomes. Osteoarthritis and Cartilage, v. 16, n. 12, p. 1519-1525, 2008.

11. MAY, S. A. Radiological aspects of degenerative joint disease. Equine Veterinary Education, v. 8, n. 2, p. 114-120, 1996.

12.PERSSON, L. On the synovia in horses. A clinical and experimental study. Acta Veterinaria Scandinavica Supplement, v. 35, p. 3-77, 1971. Supplementum.

13.MARANHÃO, R. P. A.; PALHARES, M. S.; MELO, U. P.; REZENDE, H. H. C.; BRAGA, C. E.; SILVA FILHO, J. M.; VASCONCELOS, M. N. F. Most frequent pathologies of the locomotor system en equids used for wagon traction in Belo Horizonte. Arquivo Brasileiro Medicina Veterinária e Zootecnia, v. 58, n. 1, p. 21-27, 2006

14.HOPPER, B. J.; STEEL, C.; RICHARDSON, J. L.; ALEXANDER, G. R.; ROBERTSON, I. D. Radiographic evaluation of sclerosis of the third carpal bone associated with exercise and the development of lameness in Standardbred races horses. Equine Veterinary Journal, v. 36, n.5, p. 441446, 2004.

15.BRUYERE, O.; GENANT, H.; KOTHARI, M.; ZAIM, S.; WHITE, D.; PETERFY, C.; BURLET, N.; RICHY, F; ETHGEN, D.; MONTAGUE, T.; DABROWSKI, C.; REGINSTER, J. Y. Longitudinal study of magnetic resonance imaging and standard X-rays to assess disease progression in osteoarthritis. Osteoarthritis and Cartilage, v. 5, n. 1, p.98-103, 2007.

16. WIDMER, W. R.; BLEVINS, W. E. Radiographic evaluation of degenerative joint disease in horses: interpretive principles. The Compendium, v. 16, n. 7, p. 907-918, 1994.

17.VERSCHOOTEN, F; SCHARAMME, M. Radiological examination of the tarsus. Equine Veterinary Education, v. 6, n. 2, p. 323-332, 1994 
18.SMITH, R. K.; DYSON, S. J.; SCHRAMME, M. C.; HEAD, M. J.; PAYNE, R. J.; PLATT, D.; WALMSLEY, J. Osteoarthritis of the talocalcaneal joint in 18 horses. Equine Veterinary Journal, v. 37, n. 2, p. 166-171, 2005.
19.CANTLEY, C. E.; FIRTH, E. C.; DELAHUNT, J. W.; PFEIFFER, D. U.; THOMPSON, K. G. Naturally occurring osteoarthritis in the metacarpophalangeal joints of wild horses. Equine Veterinary Journal, v. 31, n. 1, p. 73-81, 1999. 